Research Paper

\title{
On optimization of load-balanced IP routing for hose model
}

\author{
Eiji OKI \\ The University of Electro-Communications
}

\begin{abstract}
This paper presents an optimization of IP load-balanced routing for the hose model. We present an IP load-balanced routing scheme based on the two-phase routing over shortest paths. It is called a fine two-phase routing (F-TPR) scheme. In F-TPR, traffic is distributed from a source node to intermediate nodes more finely, compared to the original TPR. F-TPR introduces the distribution ratio to node $m$ that is determined for each source-destination pair of $(p, q), k_{m}^{p q}$. To determine an optimum set of $k_{m}^{p q}$, an linear programming (LP) formulation is first derived. However, the formulation is difficult to solve as a simple LP problem. This is because each element of the traffic matrix is not determined because of the hose model and there are too many possible parameters for us to consider. By introducing a duality theorem, we successfully formulate our problem a quadratic constraint programming (QCP) formulation that can be solved to determine the split ratios by using a mathematical programming solver. We compare F-TPR with TPR and the Multi-Protocol Label Switching (MPLS)-Traffic Engineering (TE). Numerical results show that F-TPR reduces the network congestion ratio compared to TPR. Numerical results show that F-TPR greatly reduces the network congestion ratio compared to TPR, and provides comparable routing performances to that of MPLS-TE.
\end{abstract}

\section{KEYWORDS}

Optimization, IP network, load-balancing, routing

\section{Introduction}

Adopting an appropriate routing scheme can increase the network resource utilization rate and network throughput of Internet Protocol (IP) networks [1]. Since it optimizes the assignment of traffic resources, additional traffic can be supported. One useful approach to enhancing routing performance is to minimize the maximum link utilization rate, also called the network congestion ratio, of all network links. Minimizing the network congestion ratio leads to increasing additional admissible traffic.

Several routing strategies have been extensively studied [2]-[9]. Wang et al. [2] formulates a general traf-

Received February 9, 2010; Revised October 29, 2010; Accepted November 4, 2010.

oki@ice.uec.ac.jp

* This work was supported in part by the Okawa Foundation and the Support Center for Advanced Telecommunications Technology Research (SCAT). DOI: $10.2201 / \mathrm{NiiPi} .2011 .8 .12$ fic engineering problem, where traffic demands are assumed to be flexibly split among source and destination nodes. This sophisticated operation is performed by the Multi-Protocol Label Switching (MPLS) TrafficEngineering (TE) technology [10]. However, legacy networks mainly employ shortest-path-based routing protocols such as Open Shortest Path First (OSPF) and Intermediate System to Intermediate System (IS-IS). This means that already deployed IP routers in the legacy networks need to be upgraded, which significantly increases capital expenditures. Therefore, it is desirable that an existing IP routing protocol still in use should be utilized.

Traffic engineering schemes that set optimum link weights in OSPF-based networks were addressed in [3]-[5]. When traffic demands are changed, optimum link weights are re-calculated and network oper- 
ators configure the updated link weights. According to the updated weights, IP routes are changed. Changing routes frequently causes network instability, which leads to packet loss and the formation of loops.

Load-balanced routing increases network resource utilization efficiency [1], [6], [7], under given traffic conditions. It is difficult for network operators to measure and predict the actual traffic matrix [5], [9]. The traffic matrix is denoted as $T=\left\{d_{p q}\right\} . d_{p q}$ is a traffic demand between source node $p$ and destination node $q$. It is, however, easy for network operators to specify the traffic as just the total outgoing/incoming traffic from/to node $p$ and node $q$, The total outgoing traffic from node $p$ is represented as $\sum_{q} d_{p q}=\alpha_{p}$, where $\alpha_{p}$ is the traffic that node $p$ can send into the network. The total incoming traffic to node $q$ is represented as $\sum_{p} d_{p q}=\beta_{q}$, where $\beta_{q}$ is the traffic that node $q$ can receive from the network. The traffic model that is specified by the total outgoing and incoming traffic for the network is called by the hose model [11]-[13].

Oki et al. presented a simple shortest-path-based load-balanced IP routing scheme for the hose model [14], which is an extension of the Smart-OSPF scheme [6]. The extended S-OSPF scheme, the same as the original S-OSPF, splits traffic demand only at source edge nodes and transmits the traffic along the shortest path routes. In the original S-OSPF scheme, the split ratios are determined for each source-destination edge node pair by assuming that the traffic demand between all source-destination edge node pairs are known, in other words, the exact traffic matrix is completely given. On the other hand, extended S-OSPF assumes the use of the hose model. Extended S-OSPF enhances the routing performance compared with the classical shortest path routing scheme. However, since source edge nodes distribute traffic only to the neighbor nodes, the routing performance of S-OSPF highly depends on the number of neighbor nodes.

Kodialam et al. [7], [8] introduced a general twophase routing strategy for the hose model. The routing strategy performs load balancing in two stages across intermediate nodes. A linear programming problem was presented to maximize the network throughput to determine routing between source and intermediate nodes and between intermediate and destination nodes. Zhang et el. presents a fault-tolerant network design approach based on the two-phase routing strategy [1].

Antić et al. [9] extends the general two-phase routing strategy presented in [7] to a simpler routing strategy than the general one by using shortest paths between source and intermediate nodes and between intermediate and destination nodes for the hose model, as shown in Fig. 1. To differentiate the original twophase routing strategy presented in [7], [8] from the ex-

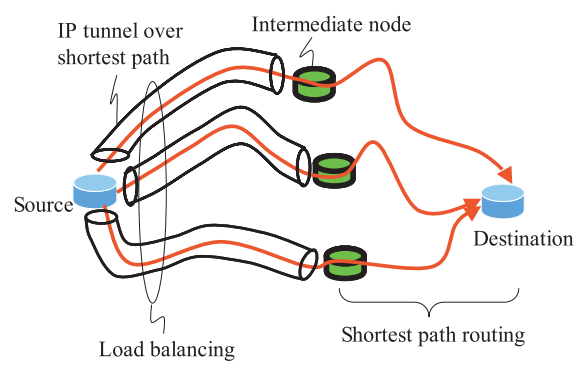

Fig. 1 Network structure of Two-Phase Routing (TPR) and Fine TPR (F-TPR) based on shortest paths.

tended routing strategy over shortest paths presented in [9], this paper calls the former strategy the original two-phase routing (TPR), and simply the latter strategy TPR. TPR performs load balancing and each flow is routed according to the OSPF protocol, which is an existing IP routing protocol, in two stages across intermediate nodes. TPR makes the number of possible routes increase for load balancing. This reduces network congestion. In TPR, however, a source node distributes traffic to all the nodes in the network as transit or destination nodes with a set of optimum distribution ratios that are set to be the same values among all source-destination pairs. The distribution ratio to node $m$ for all the source-destination pairs is $k_{m}$, where $0 \leq k_{m} \leq 1$ and $\sum_{m} k_{m}=1$. $k_{m}$ does not depend on source-destination pair of $(p, q)$. As a result, the traffic load is not efficiently distributed so as to minimize the network congestion ratio. It is also possible for some traffic to go through the same node twice on the two routes of the first and second stages.

Fig. 2 shows an example of TPR. First, consider traffic from node 1 to node 3, as shown in Fig. 2 (a). Intermediate nodes are nodes 2,3 , and 4 , but node 3 is also the destination node. In the first phase, node 1 distributes traffic to nodes 2,3 , and 4 with the distribution ratios of $k_{2}, k_{3}$, and $k_{4}$, respectively, through IP tunnels, which are routed on their shortest paths. In the second phase, intermediate nodes 2 and 4 forward traffic to node 3 on the shortest paths. As node 3 is the destination node, the second phase does not exist for node 3. Second, consider traffic from node 1 to node 2 , as shown in Fig. 2 (b). Intermediate nodes are nodes 2, 3, and 4 , but node 2 is also the destination node. In the first phase, node 1 distributes traffic to nodes 2,3 , and 4 with the same distribution ratios as the case of Fig. 2 (a). In the second phase, intermediate nodes 3 and 4 forward traffic to node 2 on the shortest paths. As node 2 is the destination node, the second phase does not exist for node 2 . The route from node 1 to node 2 via node 3 is clearly not efficient, because a link between node 2 


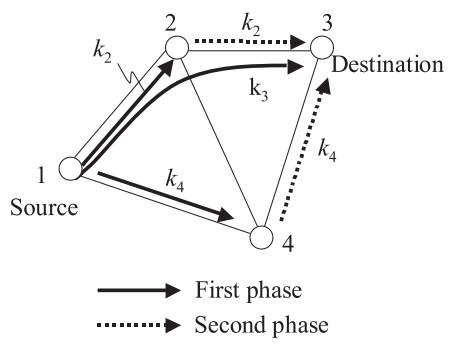

(a) Traffic from node 1 to node 3

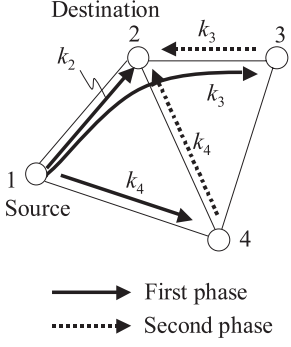

(b) Traffic from node 1 to node 2

Fig. 2 Example of TPR.

and node 3 is used twice for the same traffic in case of $k_{3} \neq 0$, where $k_{3}$ is determined considering traffic for all source and destination node pairs.

Therefore, to make traffic load efficiently balanced, the distribution ratio to node $m$ should be determined for each each source-destination pair of $(p, q)$. In other words, the distribution ratio should be defined as $k_{m}^{p q}$, where $0 \leq k_{m}^{p q} \leq 1$ and $\sum_{m} k_{m}^{p q}=1$.

This paper presents an optimization of IP loadbalanced routing for the hose model. We introduce an IP load-balanced routing scheme based on the twophase routing over shortest paths [15]. It is called a fine two-phase routing (F-TPR) scheme [16]. In [16], F-TPR was proposed assuming that the exact traffic matrix is completely given. This paper describes detail analysis of F-TPR by extending our previous work in [15], by assuming the hose model, where the total outgoing and incoming traffic to each edge node in the network is given. The network structure of F-TPR is similar to that of TPR, as shown in Fig. 1. However, in F-TPR, traffic is distributed from a source node to multiple intermediate nodes more finely, compared to TPR. F-TPR introduces the distribution ratio to node $m$ that is determined for each source-destination pair of $(p, q), k_{m}^{p q}$. To determine an optimum set of $k_{m}^{p q}$, an linear programming (LP) formulation is first derived. However, the formulation is difficult to solve as a simple LP problem. This is because each element of the traffic matrix is not determined and there are too many possible parameters for us to consider. By introducing a duality theorem [5], we successfully formulate our problem a quadratic constraint programming (QCP) formulation that can be solved to determine the split ratios by using a mathematical programming solver. We compare F-TPR with TPR and MPLS-TE. Numerical results show that F-TPR greatly reduces the network congestion ratio compared to TPR, especially when the network topology becomes dense. In addition, F-TPR provide the network congestion ratio close to that of

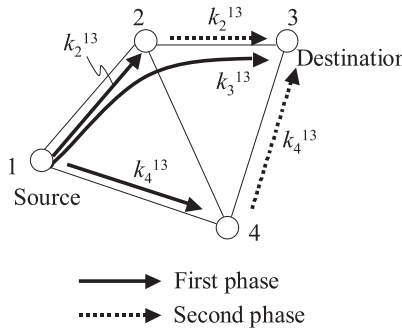

(a) Traffic from node 1 to node 3

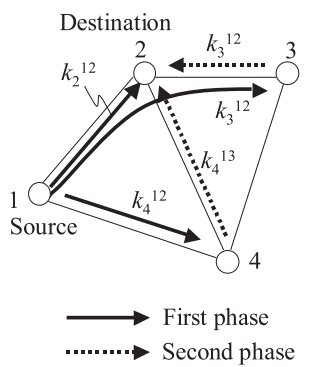

(b) Traffic from node 1 to node 2
Fig. 3 Example of F-TPR.

MPLS-TE within the difference of $10 \%$.

Fig. 3 shows an example of F-TPR. Compared with the example of TPR, as shown in Fig. 2, the distribution ratios of F-TPR are different from those of TPR. In FTPR, the distribution ratios of $k_{2}^{13}, k_{3}^{13}$, and $k_{4}^{13}$ are set for traffic from node 1 to node 3 , while those of $k_{2}^{12}, k_{3}^{12}$, and $k_{4}^{12}$ are differently set for traffic from node 1 to node 2. For traffic from node 1 to node $2, k_{3}^{12}$ is able to set to 0 in order to avoid using a link between node 2 and node 3 twice, because $k_{3}^{12}$ is independent of $k_{3}^{13}$. Thus, F-TPR solves the issue of inefficient traffic distribution.

The remainder of this paper is organized as follows. Section 2 uses a network model to introduce the terminology of this paper. Section 3 describes the original TPR scheme. Section 4 describes the proposed F-TPR scheme. Section 5 evaluates the performance of F-TPR in a comparison with TPR. Finally, Section 6 summarizes the key points.

\section{Network model}

The network is represented as a directed graph $G(V, E)$, where $V$ is the set of vertexes (nodes) and $E$ is the set of links. A link from node $i \in V$ to node $j \in V$ is denoted as $(i, j) \in E . c_{i j}$ is the capacity of $(i, j) \in E$. $L_{i j}$ is the link load of $(i, j) \in E . T=\left\{d_{p q}\right\}$ is the traffic matrix, where $d_{p q}$ is the traffic demand from node $p$ to node $q . \alpha_{p}$ is the traffic that node $p$ can send into the network. $\beta_{q}$ is the traffic that node $q$ can receive from the network. $T=\left\{d_{p q}\right\}$ is bounded by the hose model as follows.

$$
\begin{array}{ll}
\sum_{q \in V} d_{p q}=\alpha_{p} & \\
\sum_{p \in V} d_{p q}=\beta_{q} & q \in V
\end{array}
$$

Let a set of $T$ s that satisfy the condition specified by Eqs. (1a)-(1b). 
The network congestion ratio, which refers to the maximum value of all link utilization rates in the network, is denoted as $r$. Minimizing $r$ means that additional admissible traffic is maximized. The additional admissible traffic volume is accepted up to the current traffic volume multiplied by $1 / r$. Minimizing $r$ with routing control is the objective of this paper. In this case, the length of each computed path between source and destination nodes does not always choose the shortest path.

\section{Two phase routing with hose model}

In TFR [9], the traffic from node $p$ to node $q$ is not sent directly. It is split in portions that are directed to intermediate node $m \in V$. For all source-destination pairs $(p, q)$, the portion of a flow $d_{p q}$ that is balanced across a node $m$ equals $k_{m}$, where $0 \leq k_{m} \leq 1$ and $\sum_{m} k_{m}=1$. Then, every intermediate node $m$ forwards the received traffic to its final destination node $q$. Traffic from node $p$ to node $m$ and from node $m$ to node $q$ is routed along the shortest paths. TRF assumes to use an existing IP protocol, where the configuration of IP tunnels is required, such as IP-in-IP and Generic Routing Encapsulation (GRE) tunnels, between all edge nodes and intermediate nodes in the network.

We briefly review the formulation to determine an optimum set of $k_{m}$ so as to minimize the network congestion ratio, $r$, in the hose model. Consider traffic from node $p$ to node $q$ across node $m$. Let $b_{p m}$ the traffic between node $p$ and node $m$. The traffic between node $p$ and node $m$ consists of two components. The first one is the traffic generated by node $p$ and balanced across node $m$, which is defined as $b_{p m}^{(1)}$. The second one is the traffic for $m$ balanced across node $p$, which is defined as $b_{p m}^{(2)}$. Therefore, $b_{p m}$ is given by:

$$
b_{p m}=b_{p m}^{(1)}+b_{p, m}^{(2)} .
$$

The traffic generated by node $p$ and balanced across node $m$ is given by:

$$
b_{p m}^{(1)}=\sum_{q \in V} k_{m} d_{p q}=k_{m} \alpha_{p} .
$$

The traffic for $m$ balanced across node $p$ is given by:

$$
b_{p m}^{(2)}=\sum_{u \in V} k_{p} d_{u m}=k_{p} \beta_{m} .
$$

In Eqs. (3) and (4), the equalities are obtained by the constraints of the hose model. Thus,

$$
b_{p m}=k_{m} \alpha_{p}+k_{p} \beta_{m} .
$$

Let the variable $F_{p m}^{i j}$ set 1 if $(i, j)$ belongs to the shortest path between the nodes $p$ and $m$ and otherwise 0 . The link load $L_{i j}$ of $(i, j)$ is given by:

$$
\begin{aligned}
L_{i j} & =\sum_{p \in V} \sum_{m \in V} F_{p m}^{i j} b_{p m} \\
& =\sum_{p \in V} \sum_{m \in V} F_{p m}^{i j}\left(k_{m} \alpha_{p}+k_{p} \beta_{m}\right) .
\end{aligned}
$$

An optimal routing formulation with TPR for the hose model to determine the distribution ratio $k_{m}$ is as follows.

$$
\begin{aligned}
& \min r \\
& \text { t. } \sum_{m \in V} k_{m}=1 \\
& \sum_{p \in V} \sum_{m \in V} F_{p m}^{i j}\left(k_{m} \alpha_{p}+k_{p} \beta_{m}\right) \leq c_{i j} \cdot r \\
& \sum_{i \in V} \sum_{p \in V} \sum_{m \in V}\left(F_{p m}^{i j}-F_{p m}^{j i}\right)\left(k_{m} \alpha_{p}+k_{p} \beta_{m}\right) \\
& \quad=\beta_{j}-\alpha_{j} \quad j \in V \\
& 0 \leq k_{m} \leq 1 \quad m \in V \\
& 0 \leq r \leq 1
\end{aligned}
$$

The objective function in Eq. (7a) minimizes the network congestion ratio. Eq. (7b) states that the sum of $k_{m}$ over all intermediate nodes $m$ is equal to 1 . Eq. (7c) indicates that the sum of the fractions of traffic demands transmitted over $(i, j)$ is equal to or less than the network congestion ratio times the total capacity $c_{i j}$ for all links. The constraints of the hose model is incorporated in Eq. (7c) by using Eqs. (3) and (4). Eq. (7d) is a constraint for flow conservation. It states that the difference of the traffic flows incoming to node $j$ and outgoing from $j$ is equal to $\beta_{j}-\alpha_{j}$. Eqs. (7a)-(7d) are an LP problem and can be solved optimally with standard LP solver.

\section{Fine two phase routing with hose model}

In F-TPR, traffic is distributed from a source node to intermediate nodes more finely, compared to the original TPR. The distribution ratio to node $m$ for each source-destination pair of $(p, q)$ is introduced as $k_{m}^{p q}$. To determine an set of optimum $k_{m}^{p q}$ that minimizes the network congestion ratio, a general programming formulation is presented in this section.

In the same way as TPR, the traffic generated by node $p$ and balanced across node $m$ is given by:

$$
b_{p m}^{(1)}=\sum_{q \in V} k_{m}^{p q} d_{p q} .
$$

The traffic for $m$ balanced across node $p$ is given by:

$$
b_{p m}^{(2)}=\sum_{u \in V} k_{p}^{u m} d_{u m}
$$


Note that Eqs. (8) and (9) are not able to be expressed by the constraints of the hose model, as is the case of Eqs. (3) and (4). This is because $k_{m}^{p q}$ depends on node pair $(p, q)$. The link load $L_{i j}$ of $(i, j)$ is obtained as:

$$
\begin{aligned}
L_{i j} & =\sum_{p \in V} \sum_{m \in V} F_{p m}^{i j} b_{p m} \\
& =\sum_{p \in V} \sum_{m \in V} F_{p m}^{i j}\left(b_{p m}^{(1)}+b_{p m}^{(2)}\right) \\
& =\sum_{p \in V} \sum_{m \in V} F_{p m}^{i j}\left(\sum_{q \in V} k_{m}^{p q} d_{p q}+\sum_{u \in V} k_{p}^{u m} d_{u m}\right) \\
& =\sum_{p \in V} \sum_{q \in V} \sum_{m \in V}\left(F_{p m}^{i j}+F_{m q}^{i j}\right) k_{m}^{p q} d_{p q} \\
& =\sum_{p \in V} \sum_{q \in V} \varphi_{p q}^{i j} d_{p q},
\end{aligned}
$$

where $\varphi_{p q}^{i j}$ is defined as:

$$
\varphi_{p q}^{i j}=\sum_{m \in V}\left(F_{p m}^{i j}+F_{m q}^{i j}\right) k_{m}^{p q}
$$

The difference of the traffic flows incoming to node $j$ and outgoing from $j$ is given by:

$$
\begin{aligned}
& \sum_{i \in V}\left(L_{i j}-L_{j i}\right) \\
= & \sum_{i \in V}\left\{\sum_{p \in V} \sum_{q \in V} \sum_{m \in V}\left(F_{p m}^{i j}+F_{m q}^{i j}\right) k_{m}^{p q} d_{p q}\right. \\
& \left.-\sum_{p \in V} \sum_{q \in V} \sum_{m \in V}\left(F_{p m}^{j i}+F_{m q}^{j i}\right) k_{m}^{p q} d_{p q}\right\} \\
= & \sum_{p \in V} \sum_{q \in V} \sum_{i \in V} \sum_{m \in V}\left(F_{p m}^{i j}+F_{m q}^{i j}-F_{p m}^{j i}-F_{m q}^{j i}\right) k_{m}^{p q} d_{p q} \\
= & \sum_{p \in V} \sum_{q \in V} \tau_{p q}^{j} d_{p q},
\end{aligned}
$$

where $\tau_{p q}^{j}$ is defined as:

$$
\tau_{p q}^{j}=\sum_{i \in V} \sum_{m \in V}\left(F_{p m}^{i j}+F_{m q}^{i j}-F_{p m}^{j i}-F_{m q}^{j i}\right) k_{m}^{p q}
$$

Let a set of $T$ s that satisfy the condition specified by Eqs. (1a)-(1b) be $\{T\}$. Assuming that $T$ is given, the network congestion ratio, $r . K$ is represented as a threedimensional distribution-ratio matrix whose element is $k_{m}^{p q}$, or $K=\left\{k_{m}^{p q}\right\} .\{K\}$ is a set of $K$.

We would like to find the optimal routing, in other words, the optimal set of distribution ratios that minimizes $r$ for $K \in\{K\}$, and maximizes the minimal $r$ in terms of $T \in\{T\}$. This routing is called oblivious routing [7], [17].

$$
\min _{K \in\{K\}} \max _{T \in\{T\}} r
$$

To find the optimal $r$ and $K$, first an optimal routing problem to obtain $\min _{K \in\{K\}} r$ is considered, under the condition that $T \in\{T\}$ is given. Then, " $\max _{T \in\{T\}}$ " in Eq. (14) is incorporated into the problem. For a given $T \in\{T\}$, an optimal routing formulation with F-TPR for the hose model to determine the distribution ratio $k_{m}^{p q}$ is as follows.

$$
\begin{aligned}
& \min r \\
\text { s.t. } & \sum_{m \in V} k_{m}^{p q}=1 \quad p, q \in V \\
& \sum_{p \in V} \sum_{q \in V} \varphi_{p q}^{i j} d_{p q} \leq c_{i j} \cdot r \quad(i, j) \in E \\
& \sum_{p \in V} \sum_{q \in V} \tau_{p q}^{j} d_{p q}=\beta_{j}-\alpha_{j} \quad j \in V \\
& 0 \leq k_{m}^{p q} \leq 1 \quad p, q, m \in V \\
& 0 \leq r \leq 1
\end{aligned}
$$

The objective function in Eq. (15a) minimizes the network congestion ratio. Eq. (15b) states that the sum of $k_{m}^{p q}$ over all intermediate nodes $m$ for each sourcedestination node pair of $(p, q)$ is equal to 1 . Eq. (15c) indicates that the sum of the fractions of traffic demands transmitted over $(i, j)$ is equal to or less than the network congestion ratio times the total capacity $c_{i j}$ for all links. Eq. (15d) states that the difference of the traffic flows incoming to node $j$ and outgoing from $j$ is equal to $\beta_{j}-\alpha_{j}$.

Although Eqs. (15a)-(15f) can be expressed as an LP problem, it cannot be easily solved as a regular LP problem. Constraint (15c) lists every valid combination in $T=\left\{d_{p q}\right\}$ specified by Eqs. (1a)-(1b). It is impossible to solve the LP problems for all possible sets of $T=\left\{d_{p q}\right\}$. A noticeable difference between the TPR formulation in Eqs. (7a)-(7f) the F-TPR formulation in Eqs. (15a)-(15f) is as follows. The TPR formulation expresses the conditions by $\alpha_{p}$ and $\beta_{q}$, and $d_{p q}$ does not appear in the formulation. On the other hand, in the F-TPR formulation, $d_{p q}$ still remains in the constraints, because $k_{m}^{p q}$ depends on node pair $(p, q)$.

The problem of Eqs. (15a)-(15f) is solved by the following property, which is obtained by extending Chu's property [5] to F-TPR, using a duality theorem.

Property 1. $k_{m}^{p q}$ achieves congestion ratio $\leq r$ for all traffic matrices in $T=\left\{d_{p q}\right\}$ constrained by the hose model if and only if there exist non-negative parameters $\pi_{i j}(p), \lambda_{i j}(p), \xi_{i j}(p)$, and $\zeta_{i j}(p)$ for every $(i, j) \in E$ such that

i) $\sum_{p \in V} \alpha_{p} \pi_{i j}(p)+\sum_{p \in V} \beta_{p} \lambda_{i j}(p)+\sum_{p \in V}\left(\beta_{p}-\alpha_{p}\right) \xi_{i j}(p)$ $\leq c_{i j} \cdot r$ for each $(i, j) \in E$

ii) $\varphi_{p q}^{i j} \leq \pi_{i j}(p)+\lambda_{i j}(q)+\sum_{m \in V} \tau_{p q}^{m} \xi_{i j}(m)$ for each $(i, j) \in$ $E$ and every $p, q \in V$.

Property 1 is proved in Appendix A. 
Property 1 allows us to replace constraints (15c) and (15d) in Eqs. (15a)-(15f) with requirements (i)-(ii) in Property 1 , and transforms the formulation into the following.

$$
\begin{array}{ll} 
& \min r \\
\text { s.t. } & \sum_{m \in V} k_{m}^{p q}=1 \quad p, q \in V \\
& \sum_{p \in V} \alpha_{p} \pi_{i j}(p)+\sum_{p \in V} \beta_{p} \lambda_{i j}(p) \\
& +\sum_{p \in V}\left(\beta_{p}-\alpha_{p}\right) \xi_{i j}(p) \leq c_{i j} \cdot r \\
& (i, j) \in E \\
& \varphi_{p q}^{i j} \leq \pi_{i j}(p)+\lambda_{i j}(q)+\sum_{m \in V} \tau_{p q}^{m} \xi_{i j}(m) \\
& p, q \in V(i, j) \in E \\
\pi_{i j}(p), \lambda_{i j}(p), \xi_{i j}(p) \geq 0 \\
0 \leq k_{m}^{p q} \leq 1 \quad p, q \in V,(i, j) \in E \\
0 \leq r \leq 1 \quad p, q \in V
\end{array}
$$

Eqs. (15c)-(15d) are replaced by Eqs. (16c)-(16d). Property 1 eliminates $T=\left\{d_{p q}\right\}$ in Eqs. (16a)-(16g) by introducing the variables of $\pi_{i j}(p), \lambda_{i j}(p)$, and $\xi_{i j}(m)$. As $\tau_{p q}^{m}$ includes liner terms in terms of $k_{m}^{p q}$ as expressed in Eq. (13), Eq. (16d) is a quadratic constraint. Therefore, Eqs. (16a)-(16g) represent a QCP formulation that can be solved to determine the split ratios by using a mathematical programming solver.

Note that the distribution ratios, $k_{m}^{p q}$, can be computed by a path computation element (PCE), which collects the information of the network topology and the hose-model parameters of $\alpha_{p}$ and $\beta_{q}$. The computed distribution ratios are sent to nodes by PCE. Each node updates their forwarding tables according to the received distribution ratios.

\section{Performance evaluation}

The performances of the four schemes, which are FTPR, TPR, and MPLS-TE [10] are compared by solving their LP or QCP problems. The performance measure is the network congestion ratio, $r$. We use randomly generated network topologies under the condition that average node degree $D$ is satisfied for a given number of nodes $N$ and at least one path exists between every source-destination node pair. $D$ is the average number of other nodes to which individual nodes are connected by links. Link capacities are randomly generated with uniform distribution in the range of $(80,120) . \alpha_{p}$ and $\beta_{q}$, which are the parameters of the hose models, are also randomly generated in the range of $(0,100)$. In F-TPR and TPR, the link weights that

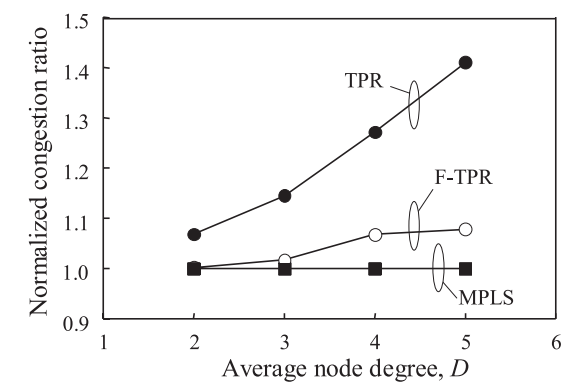

Fig. 4 Comparison of congestion ratios $(N=8)$.

are used for shortest path computation are set to be inversely proportional to the link capacities. To compare the $r$ of the different models, we normalize the network congestion ratios of F-TPR, and TPR by that of MPLSTE. The normalized network congestion ratios for FTPR, TPR, and MPLS-TE are denoted as $r_{F-T R P}, r_{T P R}$, $r_{M P L S T E}(=1.0)$ respectively. MPLS-TE provides the best routing so as to minimize the network congestion ratio due to its sophisticated operations.

Fig. 4 shows comparisons of the network congestion ratios of the three schemes. We obtained the average values of the normalized network congestion ratios for 100 randomly generated networks with $N=8$. The results indicate that F-TPR reduces the network congestion ratio, compared to TPR. The reduction effect of the network congestion ratio becomes strong as $D$ increases. For example, with $D \geq 4$, the difference between $r_{F}$ and $r_{T}$ is more than $20 \%$. This is because the routing flexibility of F-TPR is much higher than that of TPR with large $D$. The results also indicate that $r_{F}$ is close to $r_{M}$ within the difference of $6 \%$ for any $D$. This means that the F-TPR, which uses a routing protocol that is already deployed in the existing network, provides flexible routing close to that of MPLS-TE, which requires functional upgrades and sophisticated operations.

The time to solve the optimization problems are measured by using a Linux-based computer with $3.00 \mathrm{GHz}$ Intel ${ }^{\circledR}$ CoreTM2 Duo CPU E8400 and 3 GB memory. For $N=6,8,10$, and 12 with $D=3$, the average times for F-TPR are 1.084, 12.562, 131.077, and $631.606 \mathrm{sec}-$ onds, respectively, while those for TPR are equal to or less than 0.004 seconds, which is the minimum measurable time in the solver. F-TPR provides higher routing performances than TPR at the cost of the computation time. Solving the optimization problems for F-TPR is able to be applied to small-size networks. However, for large size networks, it is difficult to solve them within a practical computation time. Some heuristic algorithms for FTPR are required to be developed for large-size 
networks.

\section{Conclusions}

This paper presented an IP load-balanced routing scheme based on the two-phase routing over shortest paths for the hose model. It is called a fine two-phase routing (F-TPR) scheme. F-TPR distribute traffic from a source node to intermediate nodes more finely. FTPR introduces the distribution ratio to node $m$ that is determined for each source-destination pair of $(p, q)$, $k_{m}^{p q}$. To determine an optimum set of $k_{m}^{p q}$, we successfully formulate our problem a quadratic constraint programming (QCP) formulation that can be solved to determine the split ratios by using a mathematical programming solver. Numerical results show that F-TPR greatly reduces the network congestion ratio compared to TPR, and provides the network congestion ratio close to that of MPLS-TE within the difference of $6 \%$. It is noted that solving QCP problems for F-TPR is able to be applied to small-size networks. For largesize networks, it is difficult to solve them within a practical computation time. Therefore, some heuristic algorithms for FTPR are required to be developed for largesize networks.

\section{Acknowledgement}

The author would like to thank Ayako Iwaki for helping evaluating performances on IP routing.

\section{References}

[1] R. Zhang-Shen and N. McKeown, "Designing a FaultTolerant Network Using Valiant Load-Balancing," IEEE Infocom 2008, Apr. 2008.

[2] Y. Wang and Z. Wang, "Explicit routing algorithms for internet traffic engineering," IEEE International Conference on Computer Communications and Networks (ICCCN), 1999.

[3] Y. Wang and M. R. Ito, "Dynamics of load sensitive adaptive routing," IEEE International Conference on Communications (ICC), 2005.

[4] B. Fortz and M. Thorup, "Optimizing OSPF/IS-IS weights in a changing world," IEEE Journal on Selected Areas in Communications, vol. 20, no. 4, pp. 756-767, 2002.

[5] J. Chu and C. Lea, "Optimal Link Weights for Maximizing QoS Traffic,” IEEE ICC 2007, pp. 610-615, 2007.
[6] A. K. Mishra and A. Sahoo, "S-OSPF: A Traffic Engineering Solution for OSPF based on Best Effort Networks," IEEE Globecom 2007, pp. 1845-1849, 2007.

[7] M. Kodialam, T. V. Lakshman, J. B. Orlin and S Sengupta, "Pre-Configuring IP-over-Optical Networks to Handle Router Failures and Unpredictable Traffic," IEEE Infocom 2006, Apr. 2006.

[8] M. Kodialam, T. V. Lakshman, J. B. Orlin and S. Sengupta, "Oblivious Routing of Highly Variable Traffic in Service Overlays and IP Backbones," IEEE/ACM Trans. Networking, vol. 17, no. 2, pp. 459-472, Apr. 2009.

[9] M. Antic and A. Smiljanic, "Oblivious Routing Scheme Using Load Balancing Over Shortest Paths," IEEE ICC 2008, 2008.

[10] D. Awduche et al., "Requirements for Traffic Engineering Over MPLS," RFC 2702, Sept. 1999.

[11] A. Juttner, I. Szabo, A. Szentesi, "On bandwidth efficiency of the hose resource management model in virtual private networks," IEEE Infocom 2003, pp. 386395, Mar./Apr. 2003.

[12] N. G. Duffield, P. Goyal, A. Greenberg, P. Mishra, K K. Ramakrishnan and J. E. van der Merwe, "Resource management with hoses: point-to-cloud services for virtual private networks," IEEE/ACM Trans. on Networking, vol. 10, no. 5, pp. 679-692, Oct. 2002.

[13] A. Kumar, R. Rastogi, A. Silberschatz and B. Yener, "Algorithms for provisioning virtual private networks in the hose model," the 2001 Conference on Applications, Technologies, Architectures, and Protocols for Computer Communications, pp. 135-146, 2001.

[14] E. Oki, A. Iwaki, A. Masuda and K. Shiomoto, "Efficient Load-Balanced IP Routing Scheme Based on Shortest Paths in Hose Model," IEEE ICC 2009, June 2009.

[15] E. Oki and A. Iwaki, "F-TPR: Fine Two-Phase IP Routing Scheme over Shortest Paths for Hose Model," IEEE Commun. Letters, vol. 13, no. 4, pp. 277-279, Apr. 2009.

[16] E. Oki and A. Iwaki, "Fine Two-Phase Routing with Traffic Matrix," 18th International Conference on Computer Communications and Networks (ICCCN 2009), Aug. 2009.

[17] D. Applegate and E. Cohen, "Making routing robust to changing traffic demands: algorithms and evaluation," IEE/ACM Trans. Networking, vol. 14, no. 6, pp. 11931206, 2006. 


\section{Appendix}

Property 1. $k_{m}^{p q}$ achieves congestion ratio $\leq r$ for all traffic matrices in $T=\left\{d_{p q}\right\}$ constrained by the hose model if and only if there exist non-negative parameters $\pi_{i j}(p), \lambda_{i j}(p), \xi_{i j}(p)$, and $\zeta_{i j}(p)$ for every $(i, j) \in E$ such that

i) $\sum_{p \in V} \alpha_{p} \pi_{i j}(p)+\sum_{p \in V} \beta_{p} \lambda_{i j}(p)+\sum_{p \in V}\left(\beta_{p}-\alpha_{p}\right) \xi_{i j}(p)$ $\leq c_{i j} \cdot r$ for each $(i, j) \in E$

ii) $\varphi_{p q}^{i j} \leq \pi_{i j}(p)+\lambda_{i j}(q)+\sum_{m \in V} \tau_{p q}^{m} \xi_{i j}(m)$ for each $(i, j) \in$ $E$ and every $p, q \in V$

Proof: To prove Property 1, we extend Chu's proof to F-TPR [5].

("only if" direction): Let routing $k_{m}^{p q}$ have congestion ratio $\leq r$ for all traffic matrices constrained by the hose model. (i.e, $\sum_{p, q \in V} \varphi_{p q}^{i j} d_{p q} \leq c_{i j} \cdot r$ for all $(i, j)$ ). The problem of finding $T=\left\{d_{p q}\right\}$ that maximizes link load on $(i, j)$ is formulated as the following LP problem.

$$
\begin{aligned}
& \max \sum_{p \in V} \sum_{q \in V} \varphi_{p q}^{i j} d_{p q} \\
\text { s.t. } & \sum_{q \in V} d_{p q}=\alpha_{p} \quad p \in V \\
& \sum_{p \in V} d_{p q}=\beta_{q} \quad q \in V \\
& \sum_{p \in V} \sum_{q \in V} \tau_{p q}^{j} d_{p q}=\beta_{j}-\alpha_{j} \quad j \in V \\
& d_{p q} \geq 0
\end{aligned}
$$

The decision variables are $d_{p q}$. The given parameters are $\varphi_{p q}^{i j}, \tau_{p q}^{j}, \alpha_{p}$, and $\beta_{q}$. The dual of the LP problem in Eqs. (17a)-(17e) for $(i, j)$ is:

$$
\begin{gathered}
\min \sum_{p \in V} \alpha_{p} \pi_{i j}(p)+\sum_{p \in V} \beta_{p} \lambda_{i j}(p) \\
+\sum_{p \in V}\left(\beta_{p}-\alpha_{p}\right) \xi_{i j}(p) \\
\text { s.t. } \varphi_{p q}^{i j} \leq \pi_{i j}(p)+\lambda_{i j}(q)+\sum_{m \in V} \tau_{p q}^{m} \xi_{i j}(m) \\
p, q \in V,(i, j) \in E \\
\pi_{i j}(p), \lambda_{i j}(p), \xi_{i j}(p) \geq 0 \\
p, q \in V,(i, j) \in E .
\end{gathered}
$$

The derivation of Eqs. (18a)-(18c) is described after this proof. Because of $\sum_{p q} \varphi_{p q}^{i j} d_{p q} \leq c_{i j} \cdot r$ in Eq. (17a), the dual, $\sum_{p \in V} \alpha_{p} \pi_{i j}(p)+\sum_{p \in V} \beta_{p} \lambda_{i j}(p)+\sum_{m \in V} \tau_{p q}^{m} \xi_{i j}(m)$ in Eq. (18a), for any $(i, j)$, must have the same optical value. The optimal value in Eq. (18a) should be $\leq c_{i j} \cdot r$. Therefore, the objective function of the dual satisfies (i). Requirement (ii) is satisfied by dual problem constraint (18b).

("if" direction): Let $\varphi_{p q}^{i j}$ be a value obtained from the routing result, and $T=\left\{d_{p q}\right\}$ be any valid traffic matrix. Let $\pi_{i j}(p), \lambda_{i j}(p)$, and $\xi_{i j}(p)$ be the parameters satisfying requirements (i)-(ii). Consider $(i, j)$. From (ii) we have,

$$
\varphi_{p q}^{i j} \leq \pi_{i j}(p)+\lambda_{i j}(q)+\sum_{m \in V} \tau_{p q}^{m} \xi_{i j}(m) .
$$

Summing over all node pairs $(p, q)$, we have

$$
\begin{gathered}
\sum_{p \in V} \sum_{q \in V} \varphi_{p q}^{i j} d_{p q} \\
\leq \sum_{p \in V} \sum_{q \in V}\left[\pi_{i j}(p)+\lambda_{i j}(q)+\sum_{m \in V} \tau_{p q}^{m} \xi_{i j}(m)\right] d_{p q} \\
=\sum_{p \in V} \pi_{i j}(p) \sum_{q \in V} d_{p q}+\sum_{q \in V} \lambda_{i j}(q) \sum_{p \in V} d_{p q} \\
+\sum_{p \in V} \sum_{q \in V} \sum_{m \in V} \tau_{p q}^{m} \xi_{i j}(m) d_{p q} \\
=\sum_{p \in V} \alpha_{p} \pi_{i j}(p)+\sum_{p \in V} \beta_{p} \lambda_{i j}(p) \\
\quad+\sum_{p \in V}\left(\beta_{p}-\alpha_{p}\right) \xi_{i j}(p)
\end{gathered}
$$

The last equality is obtained by using the constraints of the flow conservation and the hose model, which are expressed in Eqs. (15d) and Eqs. (1a)-(1b), respectively. From (i), we have

$$
\begin{aligned}
& \sum_{p \in V} \sum_{q \in V} \varphi_{p q}^{i j} d_{p q} \\
= & \sum_{p \in V} \alpha_{p} \pi_{i j}(p)+\sum_{p \in V} \beta_{p} \lambda_{i j}(p) \\
& +\sum_{p \in V}\left(\beta_{p}-\alpha_{p}\right) \xi_{i j}(p) \\
\leq & c_{i j} \cdot r .
\end{aligned}
$$

This indicates that for any traffic matrices constrained by the hose model, the load on any link is at most $r$.

Eqs. (17a)-(17e), which is the LP problem of finding $\boldsymbol{T}=\left\{d_{p q}\right\}$ that maximizes link load on $(i, j)$ is represented with a matrix expression by

$$
\begin{gathered}
\max \boldsymbol{\Phi}_{i j}^{T} \boldsymbol{d} \\
\text { s.t. } \boldsymbol{A d}=\boldsymbol{C} \\
\boldsymbol{d} \geq 0,
\end{gathered}
$$

where

$$
\begin{aligned}
\boldsymbol{\Phi}_{i j}^{T} & =\left[\varphi_{11}^{i j} \varphi_{12}^{i j} \cdots \varphi_{1 N}^{i j}|\cdots| \varphi_{N 1}^{i j} \varphi_{N 2}^{i j} \cdots \varphi_{N N}^{i j}\right] \\
\boldsymbol{d}^{T} & =\left[d_{11} d_{12} \cdots d_{1 N}|\cdots| d_{N 1} d_{N 2} \cdots d_{N N}\right]
\end{aligned}
$$




$$
\boldsymbol{A}=\left[\begin{array}{cccc|cccc|cccc|c|cccc}
1 & 1 & \cdots & 1 & 0 & 0 & \cdots & 0 & 0 & 0 & \cdots & 0 & \cdots & 0 & 0 & \cdots & 0 \\
0 & 0 & \cdots & 0 & 1 & 1 & \cdots & 1 & 0 & 0 & \cdots & 0 & \cdots & 0 & 0 & \cdots & 0 \\
0 & 0 & \cdots & 0 & 0 & 0 & \cdots & 0 & 1 & 1 & \cdots & 1 & \cdots & 0 & 0 & \cdots & 0 \\
& & \cdots & & & & \cdots & & & & \cdots & & \cdots & & & \cdots & \\
0 & 0 & \cdots & 0 & 0 & 0 & \cdots & 0 & 0 & 0 & \cdots & 0 & \cdots & 1 & 1 & \cdots & 1 \\
\hline 1 & 0 & \cdots & 0 & 1 & 0 & \cdots & 0 & 1 & 0 & \cdots & 0 & \cdots & 1 & 0 & \cdots & 0 \\
0 & 1 & \cdots & 0 & 0 & 1 & \cdots & 0 & 0 & 1 & \cdots & 0 & \cdots & 0 & 1 & \cdots & 0 \\
0 & 0 & \cdots & 1 & 0 & 0 & \cdots & 1 & 0 & 0 & \cdots & 1 & \cdots & 0 & 0 & \cdots & 1 \\
\hline \tau_{11}^{1} & \tau_{12}^{1} & \cdots & \tau_{1 N}^{1} & \tau_{21}^{1} & \tau_{22}^{1} & \cdots & \tau_{2 N}^{1} & \tau_{31}^{1} & \tau_{32}^{1} & \cdots & \tau_{3 N}^{1} & \cdots & \tau_{N 1}^{1} & \tau_{N 2}^{1} & \cdots & \tau_{N N}^{1} \\
\tau_{11}^{2} & \tau_{12}^{2} & \cdots & \tau_{1 N}^{2} & \tau_{21}^{2} & \tau_{22}^{2} & \cdots & \tau_{2 N}^{2} & \tau_{31}^{2} & \tau_{32}^{2} & \cdots & \tau_{3 N}^{2} & \cdots & \tau_{N 1}^{2} & \tau_{N 2}^{2} & \cdots & \tau_{N N}^{2} \\
\tau_{11}^{N} & \tau_{12}^{N} & \cdots & \tau_{1 N}^{N} & \tau_{21}^{N} & \tau_{22}^{N} & \cdots & \tau_{2 N}^{N} & \tau_{31}^{N} & \tau_{32}^{N} & \cdots & \tau_{3 N}^{N} & \cdots & \tau_{N 1}^{N} & \tau_{N 2}^{N} & \cdots & \tau_{N N}^{N}
\end{array}\right]
$$$$
\boldsymbol{C}=\left[\alpha_{1} \alpha_{2} \cdots \alpha_{N}\left|\beta_{1} \beta_{2} \cdots \beta_{N}\right| \beta_{1}-\alpha_{1} \beta_{2}-\alpha_{2} \cdots \beta_{N}-\alpha_{N}\right] .
$$

$N$ is the number of nodes. $\boldsymbol{d}$ is an $N N \times 1$ matrix. $\boldsymbol{\Phi}_{i j}$ is an $N N \times 1$ matrix. $\boldsymbol{A}$ is a $3 N \times N N$ matrix. $\boldsymbol{C}$ is a $3 N \times 1$ matrix. The dual of the LP problem represented by Eqs. $(22 \mathrm{a})-(23 \mathrm{~d})$ for $(i, j)$ is

$$
\begin{gathered}
\min \boldsymbol{C}^{T} z_{i j} \\
\text { s.t. } \boldsymbol{A}^{T} \boldsymbol{z}=\boldsymbol{\Phi}_{i j} \\
z_{i j} \geq 0,
\end{gathered}
$$

where

$$
\begin{aligned}
z_{i j}^{T}= & {\left[\pi_{i j}(1) \pi_{i j}(2) \cdots \pi_{i j}(N) \mid \lambda_{i j}(1) \lambda_{i j}(2) \cdots\right.} \\
& \left.\lambda_{i j}(N) \mid \xi_{i j}(1) \xi_{i j}(2) \cdots \xi_{i j}(N)\right] .
\end{aligned}
$$

$z_{i j}$ is a $3 N \times 1$ matrix. Eqs. (24a)-(25a) and Eqs. (23a)(23d) is a matrix expression of Eqs. (18a)-(18c).

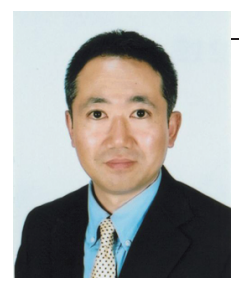

\section{Eiji OKI}

Eiji OKI is an Associate Professor of The University of ElectroCommunications, Tokyo Japan. $\mathrm{He}$ received B.E. and M.E. degrees in Instrumentation Engineering and a Ph.D. degree in Electrical Engineering from Keio University, Yokohama, Japan, in 1991, 1993, and 1999, respectively. In 1993, he joined Nippon Telegraph and Telephone Corporation's (NTT's) Communication Switching Laboratories, Tokyo Japan. He has been researching IP and optical network architectures, traffic-control methods, high-speed switching systems, and communications protocols. From 2000 to 2001, he was a Visiting Scholar at Polytechnic University, Brooklyn, New York, where he was involved in designing tera-bit switch/router systems. He joined The University of Electro-Communications, Tokyo Japan, in July 2008. Dr. Oki was the recipient of the 1998 Switching System Research Award and the 1999 Excellent Paper Award presented by IEICE, and the 2001 Asia-Pacific Outstanding Young Researcher Award presented by IEEE Communications Society for his contribution to broadband network, ATM, and optical IP technologies. He co-authored two books, "Broadband Packet Switching Technologies," published by John Wiley, New York, in 2001 and "GMPLS Technologies," published by RC Press, Boca Raton, in 2005. 